\title{
A New Insight into the Convective Boundary Condition
}

\author{
Eugen Magyari
}

Received: 8 April 2013 / Accepted: 6 May 2013 / Published online: 17 May 2013

(C) Springer Science+Business Media Dordrecht 2013

\begin{abstract}
The steady mixed convection boundary layer flows over a vertical surface adjacent to a Darcy porous medium and subject respectively to (i) a prescribed constant wall temperature, (ii) a prescribed variable heat flux, $q_{\mathrm{w}}=q_{0} x^{-1 / 2}$, and (iii) a convective boundary condition are compared to each other in this article. It is shown that, in the characteristic plane spanned by the dimensionless flow velocity at the wall $f^{\prime}(0) \equiv \lambda$ and the dimensionless wall shear stress $f^{\prime \prime}(0) \equiv S$, every solution $(\lambda, S)$ of one of these three flow problems at the same time is also a solution of the other two ones. There also turns out that with respect to the governing mixed convection and surface heat transfer parameters $\varepsilon$ and $\gamma$, every solution $(\lambda, S)$ of the flow problem (iii) is infinitely degenerate. Specifically, to the very same flow solution $(\lambda, S)$ there corresponds a whole continuous set of values of $\varepsilon$ and $\gamma$ which satisfy the equation $S=-\gamma(1+\varepsilon-\lambda)$. For the temperature solutions, however, the infinite degeneracy of the velocity solutions becomes lifted. These and further outstanding features of the convective problem (iii) are discussed in the article in some detail.
\end{abstract}

Keywords Darcy porous media - Mixed convection - Vertical surface · Convective boundary conditions $\cdot$ Self-similar solutions

\section{Introduction and Problem Formulation}

The aim of this article is to compare the solution domains of three boundary value problems to each other. The three problems are governed by the same differential equation, the celebrated Blasius equation,

$$
f^{\prime \prime \prime}+f f^{\prime \prime}=0
$$

E. Magyari $(\varangle)$

Departement Physik, Theoretische Physik, Universität Basel,

Klingelbergstrasse 82, 4056 Basel, Switzerland

e-mail: magyari@bluewin.ch 
which is subject successively to three different sets of the boundary conditions, namely

$$
\begin{aligned}
& f(0)=0, f^{\prime}(0)=1+\varepsilon_{1}, f^{\prime}(\infty)=1, \\
& f(0)=0, f^{\prime \prime}(0)=-\varepsilon_{2}, f^{\prime}(\infty)=1, \\
& f(0)=0, f^{\prime \prime}(0)=-\gamma\left[1+\varepsilon_{3}-f^{\prime}(0)\right], f^{\prime}(\infty)=1 .
\end{aligned}
$$

All the three boundary value problems $(1,2),(1,3)$, and $(1,4)$ can be associated with the very same self-similar porous flow and heat transfer problem, namely with the steady mixed convection boundary layer flow over a vertical surface adjacent to a porous medium, governed by the continuity, Darcy-Boussinesq, and energy equations (see, e.g., Merkin 1980)

$$
\frac{\partial u}{\partial x}+\frac{\partial v}{\partial y}=0, \quad u=U_{\infty}+\frac{g K \beta}{v}\left(T-T_{\infty}\right), \quad u \frac{\partial T}{\partial x}+v \frac{\partial T}{\partial y}=\alpha_{m} \frac{\partial^{2} T}{\partial y^{2}} .
$$

and subject respectively to the boundary conditions

$$
\begin{aligned}
& v=0, T=T_{\mathrm{w}} \quad \text { on } \mathrm{y}=0, \quad \text { and } \mathrm{T} \rightarrow \mathrm{T}_{\infty} \text { as } \mathrm{y} \rightarrow \infty, \\
& v=0,-k \frac{\partial T}{\partial y}=q_{0} x^{-1 / 2} \text { on } \mathrm{y}=0, \text { and } \mathrm{T} \rightarrow \mathrm{T}_{\infty} \text { as } \mathrm{y} \rightarrow \infty, \\
& v=0,-k_{\mathrm{s}} \frac{\partial T}{\partial y}=h_{0} x^{-1 / 2}\left(T_{\mathrm{f}}-T\right) \quad \text { on } \mathrm{y}=0, \text { and } \mathrm{T} \rightarrow \mathrm{T}_{\infty} \text { as } \mathrm{y} \rightarrow \infty .
\end{aligned}
$$

The boundary value problems $(1,2)$ and $(1,3)$ correspond to the mixed convection problems $(5,6)$ and $(5,7)$ with prescribed constant wall temperature $T_{\mathrm{w}}$ and prescribed variable heat flux $q_{\mathrm{w}}=q_{0} x^{-1 / 2}$ boundary conditions, respectively. Equations $(1,4)$ describe the same problem (5), but subject to the convective boundary condition given under Eq. (8).

The convective problem $(5,8)$ has comprehensively been investigated in a recent article of Lok et al. (2013) which is the basic reference for our present study. In Eqs. (5) and (8), $T$ stands for the temperature field of the fluid which saturates the porous medium and $T_{\infty}$ is its ambient temperature. The vertical surface is heated in this case by convection from an outer fluid of temperature $T_{\mathrm{f}}$. To achieve a similarity reduction of the problem, Lok et al. (2013) have assumed that the surface heat transfer coefficient $h_{\mathrm{f}}$ depends on the wall coordinate $x$ in the form $h_{\mathrm{f}}(x)=h_{0} x^{-1 / 2}$ (see the second Eq. (8)). The special form $q_{\mathrm{w}}=q_{0} x^{-1 / 2}$ of the wall heat flux in our second Eq. (7) has been chosen on the same reason.

All the three dimensionless boundary value problems can be obtained from their dimensional counterparts by the very same (Blasius-type) similarity transformations which in turn coincide formally with those used by Lok et al. (2013) in the convective case,

$$
\psi=\left(2 \alpha_{m} U_{\infty} x\right)^{1 / 2} f(\eta), \quad T=T_{\infty}+\Delta T \theta(\eta), \quad \eta=y\left(\frac{U_{\infty}}{2 \alpha_{m} x}\right)^{1 / 2},
$$

where $\psi$ is the stream function defined by $u=\partial \psi / \partial y, v=-\partial \psi / \partial x$. In Eqs. (1)-(4), the primes denote differentiations of the dimensionless stream function $f=f(\eta)$ with respect to the similarity independent variable $\eta$. Once the self-similar flow problems $(1,2)-(1.4)$ have been solved, the respective dimensionless temperature fields $\theta(\eta)=\left(T-T_{\infty}\right) / \Delta T$ are obtained (except the forced convection case $f=\eta$ ) as

$$
\theta(\eta)=\frac{f^{\prime}(\eta)-1}{\varepsilon_{i}}, \quad(f \neq \eta)
$$

where $\varepsilon_{i}$ stand for the mixed convection parameters

$$
\varepsilon_{i}=\frac{g K \beta(\Delta T)_{i}}{v U_{\infty}}, \quad i=1,2,3
$$


which represent the ratio of the Darcy-Rayleigh number and the Péclet number. The difference between the $\varepsilon_{i}$ 's of the three cases consists of the expression of $\Delta T$ which always stands for the natural temperature scale of the problem and which is $(\Delta T)_{1}=T_{\mathrm{W}}-T_{\infty}$ in the prescribed wall temperature case, $(\Delta T)_{2}=\left(q_{0} / k\right)\left(2 \alpha_{m} / U_{\infty}\right)^{1 / 2}$ in the prescribed heat flux case, and $(\Delta T)_{3}=T_{\mathrm{f}}-T_{\infty}$ in the convective case. The second parameter present in the convective boundary condition (4) is the dimensionless surface heat transfer coefficient, the Biot number $\gamma=\left(h_{0} / k_{\mathrm{S}}\right)\left(2 \alpha_{m} / U_{\infty}\right)^{1 / 2}$, where $k_{\mathrm{S}}$ is the thermal conductivity of the surface. According to Eq. (10), the values of the velocity and the shear stress at the wall, $f^{\prime}(0)$ and $f^{\prime \prime}(0)$, occurring in the boundary conditions (2)-(4) are related to the respective wall temperature and wall temperature gradient by the relationships $\theta(0)=\left(f^{\prime}(0)-1\right) / \varepsilon_{i}$ and $\theta^{\prime}(0)=f^{\prime \prime}(0) / \varepsilon_{i}$. Thus, in the prescribed wall temperature and prescribed heat flux cases (2) and (3), we recover the familiar thermal boundary conditions $\theta(0)=1$ and $\theta^{\prime}(0)=-1$, respectively, while in the convective case the linear relationship $\theta^{\prime}(0)=-\gamma[1-\theta(0)]$ is obtained. The similar temperature field $\theta(\eta)$ satisfies in all three cases the equation $\theta^{\prime \prime}+f \theta^{\prime}=0$ and the asymptotic condition $\theta(\infty)=0$. We emphasize again that in the forced convection case $f=\eta$ the relationship (10) does not exist, but the solution of the corresponding temperature equation $\theta^{\prime \prime}+\eta \theta^{\prime}=0$ can be given in all three cases in terms of the error function. These well-known cases will not be addressed in this article.

The boundary conditions (6) and (7) are quite familiar for the heat transfer community. The same holds for the convective boundary condition (8) in connection with convective heating or cooling of solids by an ambient fluid. The heat flow has to overcome in this case a surface thermal resistance characterized by the Biot number. In this respect, the convective boundary condition, called also Newton's law of cooling, is a very old issue of the heat conduction. However, in convective heat transfer in clear fluids and in porous media, the effect of the convective boundary condition (8) has attracted a significant research interest only in the latter years. Some of the representative papers in this field are those of Cortell Bataller (2008), Aziz (2009), Ishak (2010), Makinde and Olanrewaju (2010), Magyari (2011), Merkin and Pop (2011), Makinde and Aziz (2010), Uddin et al. (2012), and Lok et al. (2013).

A simple inspection of the boundary conditions (2)-(4) shows that in the limiting case $\gamma \rightarrow$ $\infty$ the convective condition (4) reduces to the prescribed wall temperature condition (2) (with $(\Delta T)_{3}$ replaced by $\left.(\Delta T)_{1}\right)$. Similarly, the prescribed heat flux condition (3) can be recovered by letting in the convective condition (4) $\gamma \rightarrow 0$ and $\varepsilon_{3} \rightarrow \infty$ simultaneously, so that $\gamma \varepsilon_{3}=$ finite $=\varepsilon_{2}$. This article emphasizes, however, that the relationship between the three boundary value problems is much closer than their punctual coincidence in the mentioned limiting cases. Namely, it will be shown that in the characteristic plane $\left(f^{\prime}(0), f^{\prime \prime}(0)\right)$ spanned by the velocity and the shear stress at the wall, the three problems become equivalent.

\section{Equivalence of the Three Boundary Value Problems}

The equivalence proof in the characteristic plane $\left(f^{\prime}(0), f^{\prime \prime}(0)\right)$ is based on the fact that the three flow problems $(1,2),(1,3)$, and $(1,4)$ share the one and the same mathematical core. This common core is the initial value problem

$$
\begin{aligned}
& f^{\prime \prime \prime}+f f^{\prime \prime}=0, \\
& f(0)=0, f^{\prime}(0)=\lambda, f^{\prime \prime}(0)=S,
\end{aligned}
$$

of which solution $f=f(\eta ; \lambda, S)$ is subject to the additional condition

$$
f^{\prime}(\infty ; \lambda, S)=1 \text {. }
$$




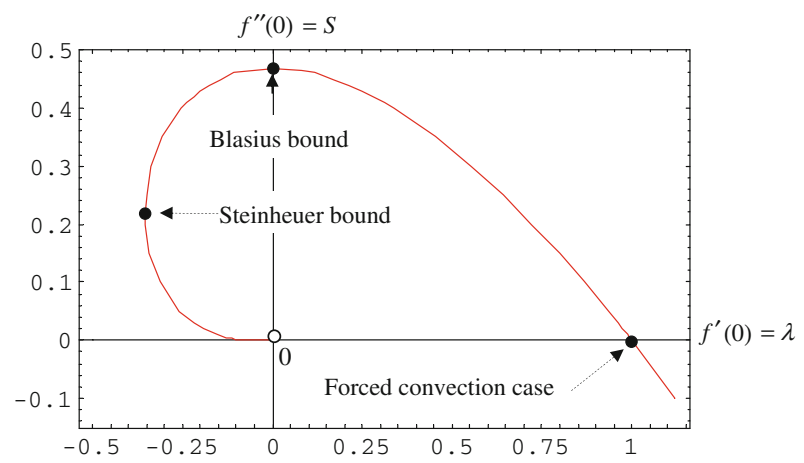

Fig. 1 Solution domain of the conditional initial value problem $(12,13)$. The origin $(\lambda, S)=(0,0)$ does not belong to the solution domain

The comfortable property of the initial value problems in comparison to the boundary value problems is that in general their solution (in the present case $f=f(\eta ; \lambda, S)$ ) exists and is unique under very mild restrictions. Thus, the existence and uniqueness of the solutions of the conditional initial value problem $(12,13)$ depends actually on whether the additional condition (13) obtained from $f=f(\eta ; \lambda, S)$ admits real solutions for $\lambda$ or $S$ at all, and whether the solutions are unique. Therefore, replacing a "boundary value problem" by a "conditional initial value problem" one transfers the difficult question of the existence and uniqueness from the differential problem to the additional condition which is either an algebraic or a transcendental equation. The solution of Eq. (13) with respect to $S$ as a function of $\lambda$ is the characteristic curve $S=S(\lambda)$ of the problem.

The determination of the characteristic curve $S=S(\lambda)$ of the conditional initial value problem $(12,13)$ is a standard numerical task and its graph obtained in this way is given in Fig. 1. The value of $S$ corresponding to $\lambda=0$ is the classical Blasius solution $S=0.4696$ which represents at the same time the upper bound of the solution domain with respect to $S$.

The lower bound of the solution domain with respect to $\lambda$ is the Steinheuer bound $(\lambda, S)=$ $(-0.3541,0.2195)$ (see below). In the range $\lambda_{\text {crit }}=-0.3541<\lambda<0$, Eq. (13) admits for every value of $\lambda$ each two solutions ("dual solutions") for $S$, i.e., in this interval $S=S(\lambda)$ is a bivalued function of $\lambda$. Furthermore, one sees that for $\lambda>0$ the solution of Eq. (13) is unique, while below the critical value $\lambda_{\text {crit }}=-0.3541$ of $\lambda$ no solutions exist at all.

During the long history of the boundary layer theory, several flow and heat transfer problems reducible to a conditional initial value problem of the form $(12,13)$ have been studied in a great detail. To these problems belongs first of all the classical Blasius problem with the no slip boundary condition $f^{\prime}(0)=0$. As it known, the solution of the Blasius problem is unique. For non-vanishing values of $\lambda$, the problem $(12,13)$ can also be associated with a boundary layer flow which is driven over a semi-infinite flat plate simultaneously by a uniform outer stream of velocity $U_{\infty}$ and the uniform motion of the plate in its own plane with velocity $U_{\mathrm{w}}$. In this case, $\lambda$ is the ratio $U_{\mathrm{w}} / U_{\infty}$ of the two velocities and can take both positive and negative values. The most frequently quoted works concerning this doubly driven Blasius flow are the articles of Klemp and Acrivos $(1972,1976)$ in which the main flow characteristics seen in Fig. 1 were reported. However, a careful scanning of the pertinent literature shows that precisely these results were obtained a few years earlier by Steinheuer (1968). Bearing in mind the uniqueness of Blasius' solution, the non-uniqueness found by Steinheuer in the range $\lambda_{\text {crit }}=-0.3541<\lambda<0$ for the doubly driven Blasius flow 
was a quite surprising result. Regrettably, Steinheuer's article (although it is quoted in the famous text of Schlichting and Gersten) remained unnoticed by the broader fluid mechanics community. In the present context of the porous media flows, the solution domain of the problem $(12,13)$ with $\lambda \equiv 1+\varepsilon$, which coincides with our problem $(1,2)$, has comprehensively been investigated by Merkin (1980). In agreement with Merkin's notation, the range $\lambda_{\text {crit }}=-0.3541<\lambda<0$ in which Steinheuer's dual solutions occur is shifted by -1 to the left to $\varepsilon_{\text {crit }}=-1.3541<\varepsilon<-1$. In the range $\varepsilon>-1$, the solutions are unique, while for $\varepsilon<\varepsilon_{\text {crit }}$, as well as for $S>0.4696$ no solutions exist. In this way, the characteristic curve $S=S(\lambda)$ seen in Fig. 1 coincides basically with that of Fig. 1 of Merkin (1980), which in turn coincides with Steinheuer's result shown in his Fig. 12 (1968). In the following, it will be assumed that the characteristic curve plotted in Fig. 1 is known also in a detailed tabular form.

Now, after the above historical remark, let us discuss the equivalence of the three boundary value problems $(1,2),(1,3)$, and $(1,4)$ in detail, considering them as special cases of the conditional initial value problem $(12,13)$.

The input parameter of the prescribed wall temperature problem $(1,2)$ is $1+\varepsilon_{1} \equiv \lambda$ and the missing boundary value which has to be determined from Eq. (13) is $S$, and this is given by the characteristic curve $S=S(\lambda)$ of Fig. 1. The prescribed heat flux problem $(1,3)$, on the other hand, is reciprocal to the problem $(1,2)$ in the sense that now the input parameter is $S \equiv-\varepsilon_{2}$ and Eq. (13) has to be solved with respect to the missing boundary value $\lambda$. This leads to a solution $\lambda=\lambda(S)$ which is nothing more than the inverse function of $S=S(\lambda)$. Therefore, mapped on the conditional initial value problem $(12,13)$ the flow problems $(1,2)$ and $(1,3)$ play entirely symmetric and equivalent roles. The one or another option is decided by the convention whether $\lambda$ or $S$ is seen as "input" or as "missing boundary value," respectively. The additional condition (13) has always to be solved with respect to the "missing boundary value" $S$ or $\lambda$. The most important consequence of the equivalent roles of $\lambda$ and $S$ in the two problems is that every solution of the problem $(1,2)$, at the same time is also a solution of the problem $(1,3)$, and conversely. More precisely, a solution $\left(f^{\prime}(0), f^{\prime \prime}(0)\right)=(\lambda, S)=\left(1+\varepsilon_{1}, S\right)$ of the prescribed wall temperature problem $(1,2)$ is at the same time a solution of the prescribed heat flux problem $(1,3)$ for $\varepsilon_{2}=-S$ and the same $f^{\prime}(0)=\lambda$. Conversely, a solution $\left(f^{\prime}(0), f^{\prime \prime}(0)\right)=\left(\lambda,-\varepsilon_{2}\right)$ of the prescribed heat flux problem $(1,3)$ is at the same time a solution of the prescribed wall temperature problem $(1,2)$ for $\varepsilon_{1}=\lambda-1$ and $f^{\prime \prime}(0)=S=-\varepsilon_{2}$.

Concerning the convective boundary value problem $(1,4)$, it is seen at the first glance that this also can be transcribed in a conditional initial value problem of the form $(12,13)$ regarding that between $\lambda$ and $S$ the relationship

$$
S=-\gamma\left(1+\varepsilon_{3}-\lambda\right)
$$

holds. Consequently, every joint solution $\left(f^{\prime}(0), f^{\prime \prime}(0)\right)=(\lambda, S)$ of the problems $(1,2)$ and $(1,3)$ corresponding to some (already determined) values of $\lambda$ and $S$ represents at the same time also a solution of the convective problem $(1,4)$ for all the parameter values $\left(\varepsilon_{3}, \gamma\right)$ which satisfy Eq. (14). That is all. The equivalence proof is completed also in this case. Therefore, at the level of consideration of the conditional initial value problem $(12,13)$, the three boundary value problems $(1,2),(1,3)$, and $(1,4)$ become basically identical. In the characteristic plane $(\lambda, S)$, the solution domain of all these three problems is specified by the very same characteristic curve $S=S(\lambda)$ drawn in Fig. 1, which thus plays the role of a "master curve" of the three problems. Although at the level of the conditional initial value problem $(12,13)$ our three boundary value problems become undistinguishable, at the level of consideration of the respective boundary conditions (2)-(4) in which the physical 


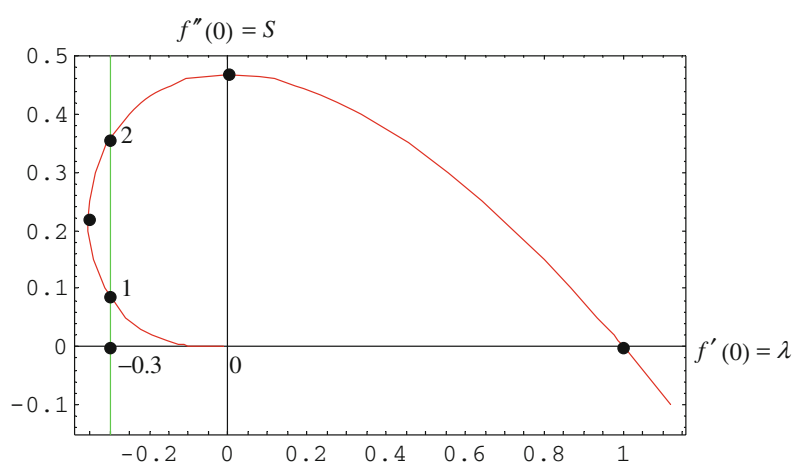

Fig. 2 The intersection points " 1 " and " 2 " of the vertical line $f^{\prime}(0)=-0.3$ with the master curve $S=S(\lambda)$ of Fig. 1 specify two solutions of the prescribed wall temperature problem $(1,2)$ corresponding to the same value $\varepsilon_{1}=-1.3$ of the mixed convection parameter

parameters $\varepsilon_{i}$ and $\gamma$ occur, between these three problems specific differences arise. It is the goal of the next sections to explain how the solution procedure can be broken down from the characteristic plane $(\lambda, S)$ to the level of the boundary conditions (2)-(4), i.e., how the individual solutions of the boundary value problems $(1,2)-(1,4)$ can be obtained with the aid of the same master curve $S=S(\lambda)$ when the values of $\varepsilon_{i}$ and $\gamma$ are specified in advance.

\section{Solution of the Problem $(1,2)$ for Specified Values of $\varepsilon_{1}$}

The solution procedure is simple and intuitive. It consists of a scanning of Fig. 1 by a vertical straight line which crosses the horizontal axis at $\lambda=1+\varepsilon_{1}$ (for details see Fig. 2).

As long as $\lambda<\lambda_{\text {crit }}=-0.3541$, i.e., $\varepsilon_{1}<\varepsilon_{1 \text {,crit }}=-1.3541$, no intersection points of the vertical line with the master curve $S=S(\lambda)$ exists and this shows once more that on the left from the Steinheuer bound no solutions of the prescribed wall temperature problem $(1,2)$ are possible. In other words, the Steinheuer bound represents the lower bound of the solution domain not only with respect to $f^{\prime}(0)$ but also with respect to the mixed convection parameter $\varepsilon_{1}$ of the problem $(1,2)$. Moving the vertical line further to the right, in the range $\varepsilon_{1 \text {,crit }}=-1.3541<\varepsilon_{1}<-1$ the dual solutions of the problem $(1,2)$ occur. To be specific, let us consider the value $\varepsilon_{1}=-1.3$, say. The (green) vertical line of Fig. 2 corresponding to $\varepsilon_{1}=-1.3$ crosses the horizontal axis at $f^{\prime}(0)=1+\varepsilon_{1}=-0.3$ and the master curve $S=S(\lambda)$ at the points marked by "1" and "2," respectively. Now, from the tabulated form of $S=S(\lambda)$ one immediately finds for the coordinates of these points, $(\lambda, S)_{1}=(-0.3,0.0849)$ and $(\lambda, S)_{2}=(-0.3,0.3566)$, respectively. These two values 0.0849 and 0.3566 of $S=f^{\prime \prime}(0)$ yield the dual solutions corresponding to $\varepsilon_{1}=-1.3$.

\section{Solution of the Problem $(1,3)$ for a Specified Value of $\varepsilon_{2}$}

Bearing in mind that with respect to the conditional initial value problem $(12,13)$ the prescribed wall temperature and the prescribed heat flux problems $(1,2)$ and $(1,3)$ are reciprocal to each other, the solution of the latter problem for a given value of the mixed convection parameter $\varepsilon_{2}$ can be obtained by a scanning of Fig. 1 by the horizontal line $f^{\prime \prime}(0)=-\varepsilon_{2}$. 


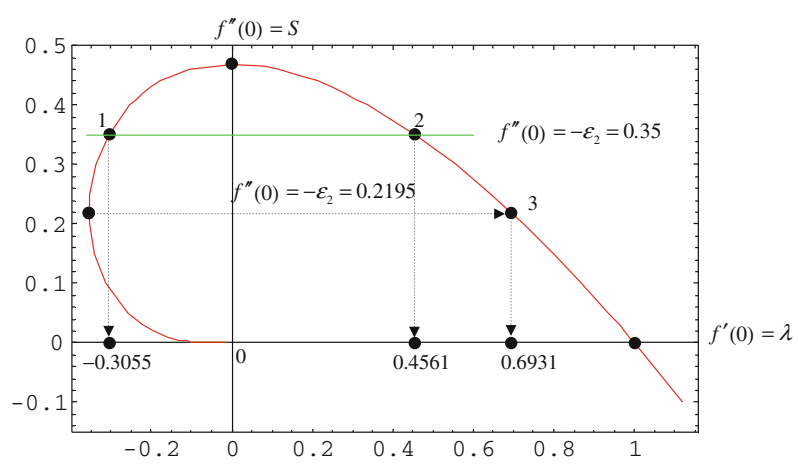

Fig. 3 Two solutions "1" and "2" of the prescribed heat flux problem $(1,3)$ corresponding to the same value $\varepsilon_{2}=-0.35$ of the input parameter. The point " 3 " is the dual counterpart of the coincident solutions corresponding to the Steinheuer bound

To be specific, let us first consider the case $\varepsilon_{2}=-0.35$, say. The solution procedure is similar to that described in Sect. 3 and is illustrated in this case in Fig. 3. It is seen that the (green) horizontal line $f^{\prime \prime}(0)=-\varepsilon_{2}=0.35$ corresponding to the selected value of $\varepsilon_{2}$ intersects the characteristic curve $S=S(\lambda)$ in two points, "1" and "2," which thus represent two solutions of the prescribed heat flux problem $(1,3)$ for $f^{\prime \prime}(0)=0.35$. From the tabulated form of the characteristic curve one finds the "coordinates" $(\lambda, S)_{1}=(-0.3055,0.35)$ and $(\lambda, S)_{2}=(0.4561,0.35)$ of the mentioned two points. The above procedure also reveals that in the range $0<f^{\prime \prime}(0)=-\varepsilon_{2}<0.4696$, i.e., $-0.4696<\varepsilon_{2}<0$ the problem (1.3) admits dual solutions, in the range $f^{\prime \prime}(0)=-\varepsilon_{2}<0$, i.e., $\varepsilon_{2}>0$ the solutions are unique, while in the range $f^{\prime \prime}(0)=-\varepsilon_{2}>0.4696$, i.e., $\varepsilon_{2}<-0.4696$ no solutions exist at all. Therefore, $\varepsilon_{2 \text {,crit }}=-0.4696$ represents in this case the lower bound of the solution domain with respect $\varepsilon_{2}$ and, at the same time the upper bound with respect to $f^{\prime \prime}(0)$. It is also interesting to notice what happens when the (green) horizontal line intersects the characteristic curve $S=S(\lambda)$ precisely at the Steinheuer bound, i.e., when $f^{\prime \prime}(0)=-\varepsilon_{2}=0.2195$ would be chosen. In this case, in addition to the two coincident solutions corresponding to the Steinheuer bound $(\lambda, S)=(-0.3541,0.2195)$, a further solution occurs at $(\lambda, S)=(0.6931,0.2195)$ (point "3" of Fig. 3).

\section{Solution of the Problem $(1,4)$ for Specified $\left(\gamma, \varepsilon_{3}\right)$}

As we have seen above, to every point $(\lambda, S)$ of the characteristic curve $S=S(\lambda)$ of Fig. 1 there corresponds the unique value $\varepsilon_{1}=\lambda-1$ of the mixed convection parameter of the problem $(1,2)$ and the unique value $\varepsilon_{2}=-S$ of the mixed convection parameter of the problem $(1,3)$. This means that these flow states $(\lambda, S)$ are non-degenerate with respect to the mixed convection parameters $\varepsilon_{1}$ and $\varepsilon_{2}$. Obviously, the converse statement is true only for $\varepsilon_{1}>-1$ and $\varepsilon_{2}>0$, respectively. In case of the convective problem $(1,4)$, however, the correspondence between the points $(\lambda, S)$ of the characteristic curve $S=S(\lambda)$ and the pertinent parameters $\gamma$ and $\varepsilon_{3}$ is basically different. Namely, the flow states $(\lambda, S)$ of the convective problem are not associated with unique values of $\gamma$ and $\varepsilon_{3}$, but with the continuous infinity of values $\left(\gamma, \varepsilon_{3}\right)$ which satisfy Eq. (14). Let us first illustrate this basic feature by means of an example. With this aim, we consider the joint solution $(\lambda, S)=(0.6931,0.2195)$ of the problems $(1,2)$ and $(1,3)$ marked by the point " 3 " of Fig. 3. According to the above 


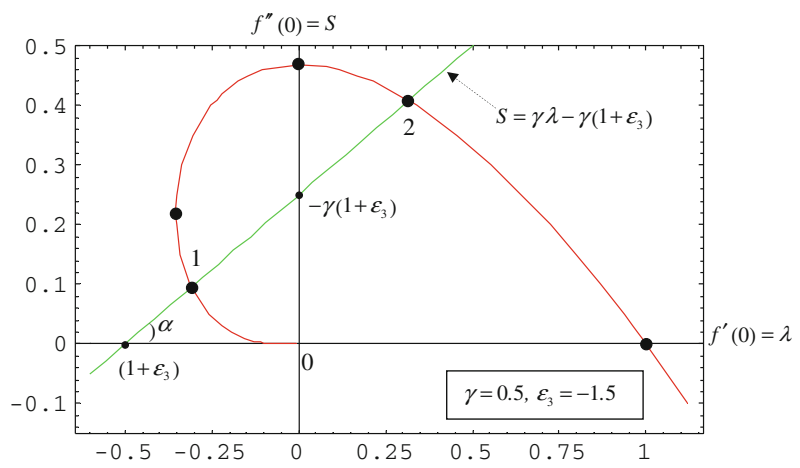

Fig. 4 The coordinates of the intersection points of the straight line (15) with the characteristic curve are $\left(\lambda_{1}, S_{1}\right)=(-0.3093,0.0953)$ and $\left(\lambda_{2}, S_{2}\right)=(0.31597,0.40798)$, respectively

arguments, this $(\lambda, S)$ is also a solution of the convective problem $(1,4)$ for the continuous set of the parameter values $\left(\gamma, \varepsilon_{3}\right)$ which satisfy Eq. (14) and which in this case becomes $0.21951=-\gamma\left(1+\varepsilon_{3}-0.69313\right)$. Obviously, there are infinity of such values $\left(\gamma, \varepsilon_{3}\right)$, and this means that the flow state $(\lambda, S)=(0.6931,0.2195)$ of the convective problem is infinitely degenerate with respect to the parameters $\gamma$ and $\varepsilon_{3}$.

Now, after the degeneracy of the convective states has been evidenced, let us explain how in this case the straightforward solution procedure works, i.e., how the solution(s) of the problem $(1,4)$ can be obtained from the same master curve $S=S(\lambda)$ of Fig. 1 for some values of the parameters $\gamma$ and $\varepsilon_{3}$ which are given in advance. The approach is simple and intuitive also in this case. To be specific, let us first consider the case $\left(\gamma, \varepsilon_{3}\right)=(0.5,-1.5)$, say. In the coordinate plane $(\lambda, S)$ of Fig. 1 , Eq. (14) is represented now by the straight line

$$
S=\gamma \lambda-\gamma\left(1+\varepsilon_{3}\right)=0.5 \lambda+0.25
$$

of which inclination angle with respect to the horizontal axis is $\alpha=\arctan (\gamma)=26.56^{\circ}$, and which intersects the characteristic curve in two points (see the green oblique line of Fig. 4). Thus, we see that the convective problem $(1,4)$ admits for $\gamma=0.5$ and $\varepsilon_{3}=-1.5$ dual solutions which are marked by the mentioned intersection points " 1 " and " 2 ." Bearing in mind Eq. (15), the coordinates of the intersection points can be determined by the very same numerical procedure as that used for the characteristic curve $S=S(\lambda)$ of Fig. 1 . In this way, we obtain for coordinates of the points " 1 " and " 2 " $\left(\lambda_{1}, S_{1}\right)=(-0.3093,0.0953)$ and $\left(\lambda_{2}, S_{2}\right)=(0.31597,0.40798)$, respectively. Therefore, these two points of the master curve $S=S(\lambda)$ specify two solutions of the convective problem $(1,4)$ for $\varepsilon_{3}=-1.5$ and $\gamma=0.5$. However, the infinite degeneracy of all the convective flow states $(\lambda, S)$ implies that $\left(\lambda_{1}, S_{1}\right)$ and $\left(\lambda_{2}, S_{2}\right)$ specify solutions of the problem $(1,4)$ not only for the assumed parameter values $\left(\gamma, \varepsilon_{3}\right)=(0.5,-1.5)$ but also for the whole continuous set of values of $\left(\gamma, \varepsilon_{3}\right)$ which satisfy the equations

$$
0.0953=-\gamma\left(1+\varepsilon_{3}+0.3093\right) \text { and } 0.40798=-\gamma\left(1+\varepsilon_{3}-0.31597\right),
$$

respectively. It is obvious that each of the latter equations admits a non-denumerable infinity of solutions $\left(\gamma, \varepsilon_{3}\right)$, in addition to the assumed one $\left(\gamma, \varepsilon_{3}\right)=(0.5,-1.5)$ which is merely a common solution of Eq. (16). It is also clear that this feature holds for all the points $(\lambda, S) \neq(1,0)$ of the master curve of Fig. 1 and implies in turn that the continuous infinity of the parameter values $\left(\gamma, \varepsilon_{3}\right)$ which for the specified flow state $(\lambda, S)$ satisfy the relationship 


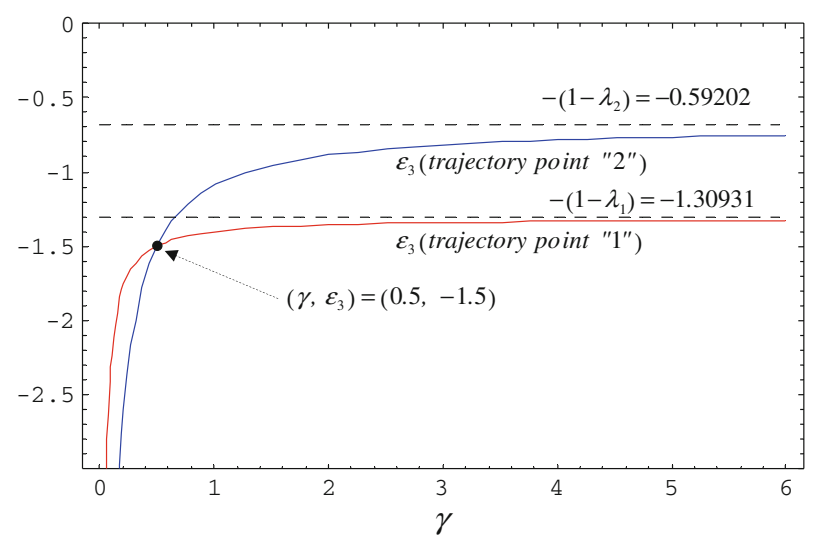

Fig. 5 The red and blue curves $\varepsilon_{3}(\gamma)$ associated with the points $\left(\lambda_{1}, S_{1}\right)=(-0.3093,0.0953)$ and $\left(\lambda_{2}, S_{2}\right)=$ $(0.31597,0.40798)$ of the master curve $S=S(\lambda)$ plotted in Fig. 4 illustrate the infinite degeneracy of the convective flow states " 1 " and " 2 "

(14), correspond to one and the same solution of the convective problem $(1,4)$. Expressed in geometrical terms, the infinite degeneracy of every flow state $(\lambda, S)$ corresponds to the fact that through every given point $(\lambda, S)$ of the master curve $S=S(\lambda)$ an infinity of straight lines $S=\gamma \lambda-\gamma\left(1+\varepsilon_{3}\right)$ can be drawn. This further implies that with every point $(\lambda, S)$ of the master curve $S=S(\lambda)$ drawn in the plane $\left(f^{\prime}(0), f^{\prime \prime}(0)\right)=(\lambda, S)$ of Fig. 1 , there is associated the whole curve

$$
\varepsilon_{3}=-\left(1-\lambda+\frac{S}{\gamma}\right) \equiv \varepsilon_{3}(\gamma) .
$$

drawn in the parameter plane $\left(\gamma, \varepsilon_{3}\right)$. This feature of the convective flow states $(\lambda, S)$ is illustrated in Fig. 5 for the case of the points "1" and "2" marked in Fig. 4. Thus, the two curves $\varepsilon_{3}=\varepsilon_{3}(\gamma)$ of Fig. 5 represent the trajectories of the flow states $\left(\lambda_{i}, S_{i}\right), i=1,2$ in the parameter plane $\left(\gamma, \varepsilon_{3}\right)$ and include the values of $\varepsilon_{3}$ and $\gamma$ associated via Eq. (14) with the points $\left(\lambda_{i}, S_{i}\right), i=1,2$ of the characteristic curve $S=S(\lambda)$. Because the points " 1 " and " 2 " are located on the same straight line (15), the two trajectories $\varepsilon_{3}=\varepsilon_{3}(\gamma)$ intersect each other at the corresponding values $\left(\gamma, \varepsilon_{3}\right)=(0.5,-1.5)$ of the parameters $\gamma$ and $\varepsilon_{3}$. In other words, the curves $\varepsilon_{3}=\varepsilon_{3}(\gamma)$ corresponding to the points $(\lambda, S)$ of the master curve $S=S(\lambda)$ fill the parameter plane $\left(\gamma, \varepsilon_{3}\right)$ of the convective flow problem $(1,4)$ densely. The intersection points of the curves $\varepsilon_{3}=\varepsilon_{3}(\gamma)$ correspond to the dual solutions associated with the same values of $\left(\gamma, \varepsilon_{3}\right)$.

\section{Lower and Upper Bounds of the Solution Domain}

The aim of this section is a detailed discussion of further specific features of the convective problem which can be predicted already by a simple inspection of Figs. 4 and 5 .

For a fixed finite value of the Biot number $\gamma$ and different values of the mixed convection parameter $\varepsilon_{3}$, Eq. (14) furnishes a family of parallel straight lines having the inclination angle $\alpha=\arctan (\gamma)$, so that $0<\alpha<90^{\circ}$. Figure 4 shows that for $\varepsilon_{3} \geq-1$ the oblique line $S=\gamma \lambda-\gamma\left(1+\varepsilon_{3}\right)$ intersects the master curve $S=S(\lambda)$ in a single point only. In the range $\varepsilon_{3}<-1$, however, two intersection points occur which, with decreasing negative 
Table 1 Critical values of the mixed convection parameter $\varepsilon_{3}$ for 16 selected values of the Biot number $\gamma$

\begin{tabular}{llll}
\hline$\gamma$ & $\lambda$ & $S$ & $\varepsilon_{3, \text { crit }}=-\left(1-\lambda+\frac{S}{\gamma}\right)$ \\
\hline$\rightarrow 0$ (Blasius bound of $S)$ & $\rightarrow 0^{-}$ & $\rightarrow 0.4696$ & $\rightarrow-\infty$ \\
0.1 & -0.0593 & 0.4667 & -5.7266 \\
0.15 & -0.0854 & 0.4635 & -4.1752 \\
0.2 & -0.1093 & 0.4593 & -3.4056 \\
0.3 & -0.1490 & 0.4497 & -2.6469 \\
0.4 & -0.1823 & 0.4377 & -2.2762 \\
0.5 & -0.2095 & 0.4256 & -2.0606 \\
0.75 & -0.2577 & 0.3959 & -1.7856 \\
1 & -0.2872 & 0.3741 & -1.6576 \\
1.4 & -0.3134 & 0.3396 & -1.5560 \\
1.8 & -0.3275 & 0.3175 & -1.5039 \\
2 & -0.3322 & 0.3086 & -1.4865 \\
3 & -0.3439 & 0.2803 & -1.4373 \\
4 & -0.3481 & 0.2660 & -1.4146 \\
5 & -0.3503 & 0.2561 & -1.4015 \\
7 & -0.3521 & 0.2454 & -1.3872 \\
10 & -0.3531 & 0.2371 & -1.3768 \\
$\rightarrow \infty$ (Steinheuer bound of $\lambda)$ & -0.3541 & $\rightarrow 0.2195$ & -1.3541 \\
\hline
\end{tabular}

The values of $(\lambda, S)=\left(f^{\prime}(0), f^{\prime \prime}(0)\right)$ give the coordinates of the points where the straight line $S=$ $\gamma \lambda-\gamma\left(1+\varepsilon_{3}\right)$ becomes tangent to the master curve $S=S(\lambda)$

values of $\varepsilon_{3}$ move first to the left and then approach each other. Thus, for every specified $\gamma$, there exists in the range $\varepsilon_{3}<-1.3541$ a smallest value of $\varepsilon_{3}$, where the two intersection points become coincident and where the straight line becomes tangent to the master curve. In this situation in the plane $\left(\gamma, \varepsilon_{3}\right)$ the trajectories (17) of the two intersection points become overlapping. This smallest, or "critical" value of $\varepsilon_{3}$ marks the lower bound of the solution domain with respect to $\varepsilon_{3}$ for the specified value of $\gamma$, such that below this critical value $\varepsilon_{3 \text {, crit }}(\gamma)$ no solutions exist at all. The dependence of $\varepsilon_{3 \text {,crit }}$ on $\gamma$ is illustrated by some examples in Table 1 and Fig. 6. For $\gamma=0.2,0.5,1.0$, and 5.0 the data of Table 1 are in a good agreement with the respective values indicated in Fig. 1a, b of Lok et al. (2013). When $\gamma \rightarrow \infty$ the straight line $S=\gamma \lambda-\gamma\left(1+\varepsilon_{3}\right)$ becomes a vertical line at $\lambda=1+\varepsilon_{3}$, which in turn becomes a tangent of the master curve $S=S(\lambda)$ precisely at the Steinheuer bound $(\lambda, S)=(-0.3541,0.2195)$. This situation corresponds to the last line of Table 1 . When, however, $\gamma \rightarrow 0$ and simultaneously $\varepsilon_{3} \rightarrow-\infty$ so that $\gamma \varepsilon_{3}=$ finite $=\varepsilon_{2}$ the straight line $S=\gamma \lambda-\gamma\left(1+\varepsilon_{3}\right)$ becomes a horizontal line at $S=-\varepsilon_{2}$, which in turn becomes a tangent of the master curve $S=S(\lambda)$ at the Blasius bound $(\lambda, S)=(0,0.4696)$. This situation corresponds to the first line of Table 1. It is also worth emphasizing here that in the prescribed wall temperature problem (i.e., for $\gamma \rightarrow \infty$ ) the Steinheuer bound at $\varepsilon_{1}=-1.3541$ represents the lower bound of the solution domain with respect to both $\varepsilon_{1}$ and $f^{\prime}(0)=1+\varepsilon_{1}$. In the convective problem, however, where $0<\gamma<\infty$, this coincidence becomes lifted and the lower bound of $\varepsilon_{3}$ follows the trajectory seen in Fig. 6 .

Obviously, Table 1 can also be read from the right to the left so that the values of the Biot number $\gamma$ corresponding to the tangents of the master curve $S=S(\lambda)$ become the "critical 


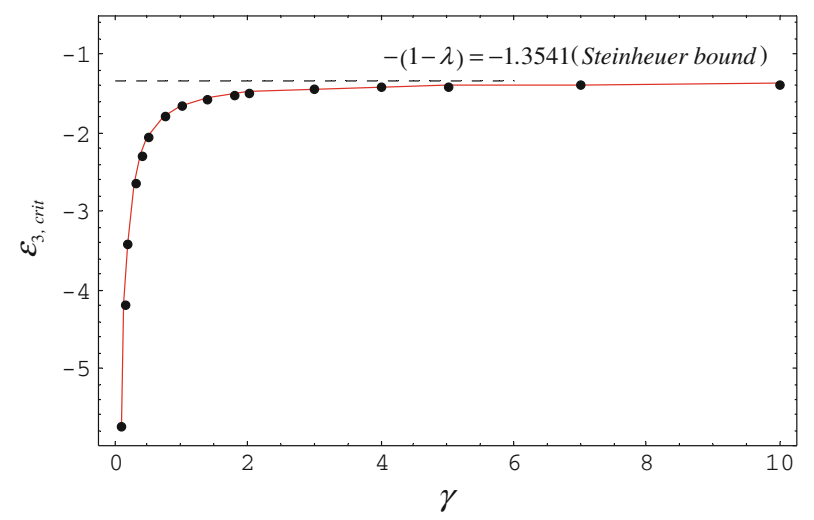

Fig. 6 Critical values of the mixed convection parameter $\varepsilon_{3}$ for the 16 selected values of the Biot number $\gamma$ are included in Table 1 . The values $\varepsilon_{3, \text { crit }}(\gamma)$ mark the lower bound of the solution domain with respect to $\varepsilon_{3}$

quantities" associated with the values of the mixed convection parameter $\varepsilon_{3}$ included in the last column of Table 1. In this sense, $\gamma_{\text {crit }}=\gamma_{\text {crit }}\left(\varepsilon_{3}\right)$ represents the upper bound of the solution domain with respect to the Biot number for the specified value of $\varepsilon_{3}$, so that for $\gamma>\gamma_{\text {crit }}\left(\varepsilon_{3}\right)$ and the same $\varepsilon_{3}$, no solutions exist. Therefore, in this respect it is plausible to speak about the critical inclination angle

$$
\alpha_{\text {crit }}\left(\varepsilon_{3}\right)=\arctan \left[\gamma_{\text {crit }}\left(\varepsilon_{3}\right)\right]
$$

of the straight line (14) which is the upper bound of the solution domain with respect to the Biot number $\gamma$ for a specified $\varepsilon_{3}$. It is also important to notice that an upper bound of $\gamma$ can only exist in the range $\varepsilon_{3}<-1.3541$ of the mixed convection parameter $\varepsilon_{3}$. Indeed, Fig. 4 shows that the oblique line $S=\gamma \lambda-\gamma\left(1+\varepsilon_{3}\right)$ can become tangent to the master curve (for $0<\gamma<\infty)$ only when its intersection point $\lambda_{0}=1+\varepsilon_{3}$ with the horizontal axis is located on the left from the Steinheuer bound, i.e., when $\lambda_{0}=1+\varepsilon_{3}<-0.3541$.

To highlight the existence of a critical Biot number $\gamma_{\text {crit }}\left(\varepsilon_{3}\right)$ related to a critical angle $\alpha_{\text {crit }}\left(\varepsilon_{3}\right)$ it is convenient to plot the "coordinates" $S=f^{\prime \prime}(0)$ and $\lambda=f^{\prime}(0)$ of the master curve $S=S(\lambda)$ of Fig. 1 as functions of $\gamma$ for specified values of the mixed convection parameter $\varepsilon_{3}$. These parametric plots are shown in Fig. 7a, b for some representative values of $\varepsilon_{3}$ namely $\varepsilon_{3}=-1.5,-1.4,-1.3$, and -1 , so that the first two values are located on the left, and the latter two on the right of the Steinheuer bound $\varepsilon_{3 \text {, crit }}=-1.3541$. The closed loops of $f^{\prime \prime}(0)$ seen in Fig. 7a, which correspond to $\varepsilon_{3}=-1.5$ and -1.4 show the existence of upper bounds of $\gamma$ for these values of $\varepsilon_{3}$. The respective values $\gamma_{\text {crit }}=1.8405$ and $\gamma_{\text {crit }}=5.1532$ associated with $f^{\prime \prime}(0)=0.3154$ and $f^{\prime \prime}(0)=0.2551$ correspond to the situations in which the oblique line (14) becomes tangent to the master curve $S=S(\lambda)$. When $\varepsilon_{3}$ increases toward the Steinheuer bound of the master curve, the loops of $f^{\prime \prime}(0)$ extend more and more to the right and $\gamma_{\text {crit }} \rightarrow \infty$ as $\varepsilon_{3} \rightarrow-1.3541$. The limiting case corresponds to the situation in which the line (14) becomes a vertical tangent to the master curve at the Steinheuer bound $(\lambda, S)=(-0.3541,0.2195)$. The same is seen in Fig. $7 \mathrm{~b}$ where the mentioned critical values of $\gamma$ correspond to $f^{\prime}(0)=-0.3286$ and $f^{\prime}(0)=-0.3505$, respectively. When, however, $\varepsilon_{3}=-1.3$, which belongs to the range $-1.3541<\varepsilon_{3}<-1$, the situation becomes basically different. Indeed, although also in this range of $\varepsilon_{3}$ dual solutions occur, the corresponding curves $f^{\prime \prime}(0)$ as functions of $\gamma$ are not closed and this means that here no upper bounds of $\gamma$ exists. The two branches of the blue curve of Fig. 7a corresponding to $\varepsilon_{3}=-1.3$ approach 

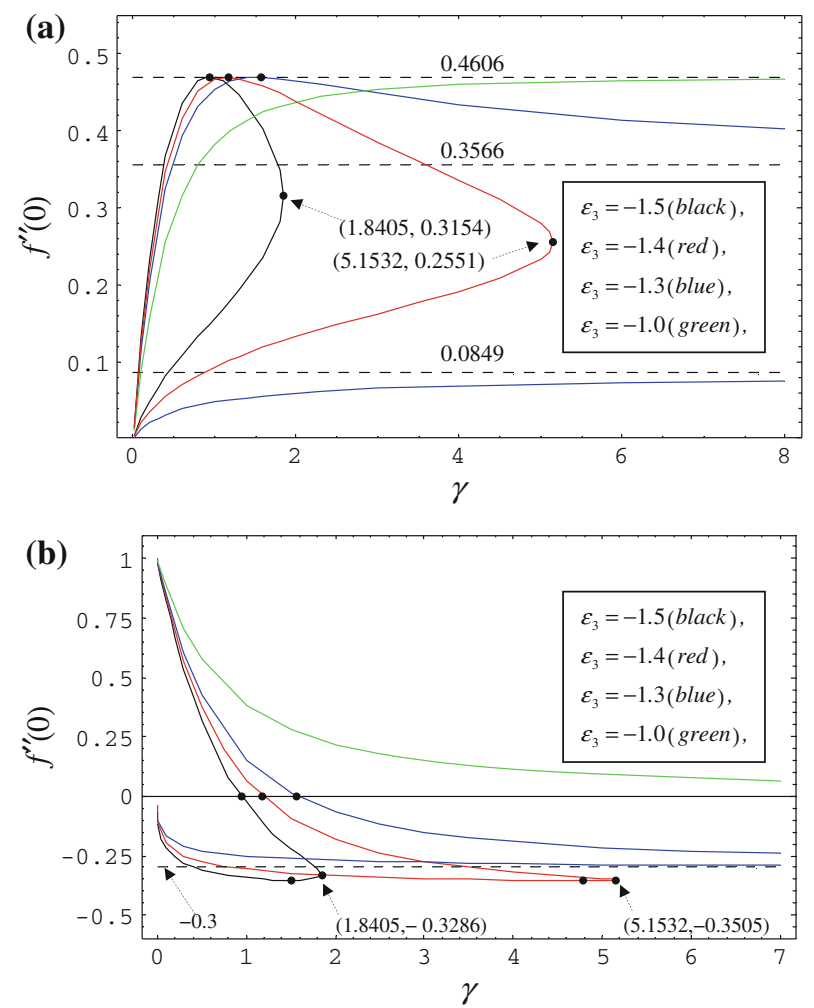

Fig. 7 The wall shear stress $f^{\prime \prime}(0)$ (upper graph) as well as the velocity at the wall $f^{\prime}(0)$ (lower graph) as functions of the Biot number $\gamma$ show a basically different behavior in the parameter ranges $\varepsilon_{3}<-1.3541$ and $-1.3541<\varepsilon_{3}<-1$. A critical Biot number $\gamma_{\text {crit }}\left(\varepsilon_{3}\right)$ as an upper bound of the solution domain with respect to $\gamma$ only exists for $\varepsilon_{3}<-1.3541$

two different asymptotes as $\gamma \rightarrow \infty$. The two asymptotes correspond precisely to the values $f^{\prime \prime}(0)=0.0849$ and $f^{\prime \prime}(0)=0.3566$ associated with the points " 1 " and " 2 " located on the vertical line $f^{\prime}(0)=-0.3$ of Fig. 2 , which in turn is the limiting case $\gamma \rightarrow \infty$ of the oblique line (14) with $\lambda=1+\varepsilon_{3}=-0.3$. The corresponding blue curves of Fig. $7 \mathrm{~b}$ both approach the horizontal asymptote $f^{\prime}(0)=-0.3$ from above as $\gamma \rightarrow \infty$, in a full agreement with Fig. 2 . The existence in the range $-1.3541<\varepsilon_{3}<-1$ of dual solutions without a critical value (upper bound) of the Biot number $\gamma$ is a remarkable feature of the convective problem $(1,4)$.

As being mentioned above, for $\varepsilon_{3} \geq-1$ the oblique line $S=\gamma \lambda-\gamma\left(1+\varepsilon_{3}\right)$ intersects the master curve $S=S(\lambda)$ in a single point only. Thus, Fig. 4 shows that $f^{\prime \prime}(0)$ is a monotonically increasing function of $\gamma$ in the range for $-1 \leq \varepsilon_{3}<0$, and a monotonically decreasing one for $\varepsilon_{3}>0$. Similarly, it is seen that $f^{\prime}(0)$ is a monotonically increasing function of $\gamma$ for $\varepsilon_{3}>0$, and a monotonically decreasing one in the range for $-1 \leq \varepsilon_{3}<0$.

This behavior is illustrated by the green curves of Fig. 7a, b. The monotonically varying functions $f^{\prime}(0)$ and $f^{\prime \prime}(0)$ approach (for $\varepsilon_{3} \geq-1$ ) certain asymptotic values which are

$$
f^{\prime}(0) \rightarrow 1+\varepsilon_{3},\left.f^{\prime \prime}(0) \rightarrow S(\lambda)\right|_{\lambda \rightarrow 1+\varepsilon_{3}} \text { as } \gamma \rightarrow \infty
$$

where $S=S(\lambda)$ is the equation of the master curve. In particular, for $\varepsilon_{3}=-1$, we obtain $f^{\prime}(0)=0, f^{\prime \prime}(0)=0.4696$ which correspond to the Blasius bound. 
In addition to the existence of critical values $\varepsilon_{3 \text {,crit }}(\gamma)$ and $\gamma_{\text {crit }}=\gamma_{\text {crit }}\left(\varepsilon_{3}\right)$ as lower and upper bounds of the solution domain in the parameter plane $\left(\gamma, \varepsilon_{3}\right)$, Fig. 4 also emphasizes further remarkable features of the boundary value problems (1)-(4). Indeed, it is seen that one never can get intersection points of the oblique line $S=\gamma \lambda-\gamma\left(1+\varepsilon_{3}\right)$ with the master curve $S=S(\lambda)$ above of the Blasius bound and on the left of the Steinheuer bound, respectively. Consequently, the Blasius bound $\left(f^{\prime}(0), f^{\prime \prime}(0)\right)=(0,0.4696)$ and the Steinheuer $\left(f^{\prime}(0), f^{\prime \prime}(0)\right)=(-0.3541,0.2195)$ represent absolute upper and lower bounds of the solution domain of all the three flow problems $(1,2),(1,3)$, and $(1,4)$ with respect to the wall shear stress $f^{\prime \prime}(0)$ and the flow velocity at the wall $f^{\prime}(0)$, respectively. These absolute bounds do not depend on the values of the mixed convection parameters $\varepsilon_{i}$ and the Biot number $\gamma$. What in the parameter plane $\left(\varepsilon_{3}, \gamma\right)$ actually happens is that the upper bound $f_{\max }^{\prime \prime}(0)=0.4696$ of $f^{\prime \prime}(0)$ and the lower bound $f_{\min }^{\prime}(0)=-0.3541$ of $f^{\prime}(0)$ migrate along certain trajectories $\varepsilon_{3}=\varepsilon_{3}(\gamma)$ which are similar to the curves seen in Fig. 5. However, during the migration the magnitudes of $f_{\max }^{\prime \prime}(0)$ and $f_{\min }^{\prime}(0)$ remain unchanged. Specifically, the two corresponding trajectories are described by equations

$$
\varepsilon_{3}(\text { Blasius bound of } S)=-\left(1+\frac{0.4696}{\gamma}\right)
$$

and

$$
\varepsilon_{3}(\text { Steinheuer bound of } \lambda)=-\left(1.3541+\frac{0.2195}{\gamma}\right),
$$

respectively. Similarly to the curves of Fig. 5, the trajectories (20) and (21) intersect each other. In this case, the coordinates of the intersection point are $\left(\gamma, \varepsilon_{3}\right)=(0.70627,-1.66490)$ and this corresponds to the situation in which the green line of Fig. 4 crosses the master curve $S=S(\lambda)$ precisely at the Steinheuer and the Blasius bound. In this case, the two bounds specify dual solutions of the convective problem $(1,4)$. The inclination angle of the mentioned straight line is $\alpha=\arctan (0.706259)=35.23^{\circ}$. For other values of $\gamma$, Eqs. (20) and (21) are associated with two different oblique lines passing the respective two bounds separately. The migration of the Blasius and the Steinheuer bounds along the trajectories (20) and (21) can qualitatively be seen also in Fig. 1a, b of Lok et al. (2013).

Solving Eq. (20) with respect to $\gamma$, we can calculate the $\gamma$ values corresponding to the migration of the Blasius bound $\left(f^{\prime}(0), f^{\prime \prime}(0)\right)=(0,0.4696)$ (i.e., to the positions of maxima of the black, red, and blue curves of Fig. 7a). Thus, we obtain $\gamma=0.9392$ for $\varepsilon_{3}=-1.5, \gamma=$ 1.1740 for $\varepsilon_{3}=-1.4$, and $\gamma=1.5653$ for $\varepsilon_{3}=-1.3$ (marked by dots on the black, red, and blue curves of Fig. 7a). Obviously, the points $\gamma=0.9392, \gamma=1.1740$, and $\gamma=1.5653$ of the $\gamma$ axis are at the same time zeros of $f^{\prime}(0)$ (plotted in Fig. 7b) for the pertinent values of $\varepsilon_{3}$. These zeros are also marked by dots on the $\gamma$ axis of Fig. 7b. Similarly, from Eq. (21), we obtain for the $\gamma$ values corresponding to the migration of the Steinheuer bound $\left(f^{\prime}(0), f^{\prime \prime}(0)\right)=(-0.3541,0.2195)$ (i.e., to the positions of minima of the black and red curves of Fig. 7b) $\gamma=1.5044$ for $\varepsilon_{3}=-1.5$ and $\gamma=4.7821$ for $\varepsilon_{3}=-1.4$ (marked by dots on the black and red curves of Fig. 7b).

\section{Temperature Solutions and Heat Transfer Characteristics}

As we have seen, the origin of the infinite degeneracy of flow states $\left(f^{\prime}(0), f^{\prime \prime}(0)\right)=(\lambda, S)$ of the convective problem $(1,4)$ resides actually in Eq. (14), $S=-\gamma\left(1+\varepsilon_{3}-\lambda\right)$, which associates with every point $(\lambda, S) \neq(1,0)$ of the master curve $S=S(\lambda)$ a continuous infinity 


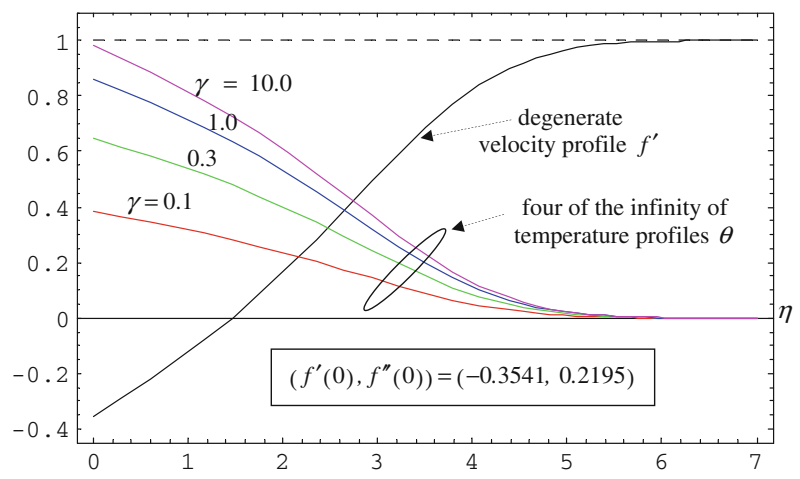

Fig. 8 The infinite degeneracy of the velocity solution $f^{\prime}(\eta)$ corresponding to the Steinheuer bound $\left(f^{\prime}(0), f^{\prime \prime}(0)\right)=(\lambda, S)=(-0.3541,0.2195)$ becomes lifted by the continuous infinity of the corresponding temperature solutions

of values $\left(\gamma, \varepsilon_{3}\right)$ which represent the trajectory $\varepsilon_{3}=\varepsilon_{3}(\gamma)$ of the flow state $(\lambda, S)$ in the plane $\left(\gamma, \varepsilon_{3}\right)$ (see, e.g., Fig. 5). This degeneracy of the flow states has important consequences on the corresponding temperature solutions and on the heat transfer characteristics of the convective boundary value problem $(1,4)$. Indeed, bearing in mind Eqs. (10) and (17) the temperature solution corresponding to a velocity solution $f^{\prime}(\eta)$ associated with a given point $(\lambda, S) \neq(1,0)$ of the master curve $S=S(\lambda)$ is

$$
\theta(\eta)=\frac{\left[1-f^{\prime}(\eta)\right] \gamma}{S+(1-\lambda) \gamma}
$$

Accordingly, the corresponding wall temperature and its gradient are obtained explicitly as

$$
\theta(0)=\frac{(1-\lambda) \gamma}{S+(1-\lambda) \gamma} \text { and } \theta^{\prime}(0)=-\frac{S \gamma}{S+(1-\lambda) \gamma},
$$

respectively. Now it is seen that Eq. (22) as a function of the Biot number $\gamma$ furnishes a continuous infinity of distinguishable temperature solutions $\theta(\eta)$, all of them being associated with the same velocity solution $f^{\prime}(\eta)$ corresponding to the very same point $(\lambda, S)$ of the master curve $S=S(\lambda)$. To every one of these temperature solutions there corresponds a unique value of $\gamma$ and, via Eq. (17), a unique value of $\varepsilon_{3}$. Therefore, for the temperature solutions (22) the infinite degeneracy of the flow solution $(\lambda, S)$ becomes lifted. In other words, while a single flow solution $(\lambda, S)$ is associated with all the points $\left(\gamma, \varepsilon_{3}\right)$ of the trajectory $\varepsilon_{3}=\varepsilon_{3}(\gamma)$ simultaneously, to every point of this trajectory there corresponds a unique temperature solution $\theta(\eta)$. This basic feature of the convective problem $(1,4)$ is illustrated in Fig. 8 for the case of a prominent flow state, namely that corresponding to the Steinheuer bound $(\lambda, S)=(-0.3541,0.2195)$.

Picking out four Biot numbers, $\gamma_{1}=0.1, \gamma_{2}=0.3, \gamma_{3}=1.0$, and $\gamma_{4}=10$, say, Eq. (17) gives with $(\lambda, S)=(-0.3541,0.2195)$ the following values of the mixed convection parameter $\varepsilon_{3,1}=-3.5492, \varepsilon_{3,2}=-2.0858, \varepsilon_{3,3}=-1.5736$, and $\varepsilon_{3,4}=-1.3760$. The infinitely degenerate velocity profile $f^{\prime}(\eta)$ corresponding to the Steinheuer bound and the mentioned four members of the continuous infinity of the associated non-degenerate temperature profiles $\theta(\eta)$ are seen in Fig. 8. From a physical and engineering point of view it is also of interest to represent the wall temperature $\theta(0)$ and the reduced Nusselt number $N u \equiv-\theta^{\prime}(0)$ as functions of the Biot number $\gamma$ according to Eq. (23), for some selected flow state $(\lambda, S)$. 


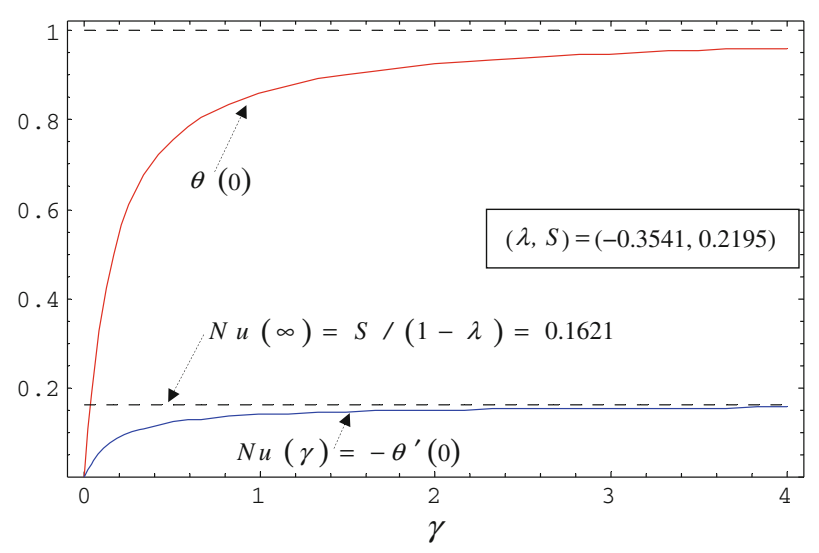

Fig. 9 Values of the wall temperature $\theta(0)$ and the reduced Nusselt number $N u(\gamma)=-\theta^{\prime}(0)$ as functions of the Biot number $\gamma$ associated the velocity solution $f^{\prime}(\eta)$ corresponding to the Steinheuer bound

This has been done in Fig. 9 for the velocity solution $f^{\prime}(\eta)$ corresponding to the Steinheuer bound $(\lambda, S)=(-0.3541,0.2195)$.

\section{Summary and Conclusions}

The effect of the convective boundary condition on the steady mixed convection boundary layer flow over a vertical surface adjacent to a Darcy porous medium has been investigated in this article on two levels of consideration. One of them is the characteristic plane $(\lambda, S)$ spanned by the dimensionless flow velocity at the wall $f^{\prime}(0) \equiv \lambda$, and the dimensionless wall shear stress $f^{\prime \prime}(0) \equiv S$. The second level of consideration is the parameter plane $\left(\gamma, \varepsilon_{3}\right)$ specified by the Biot number $\gamma$ and the mixed convection parameter $\varepsilon_{3}$. The main results of the article can be summarized as follows.

(1) The convective flow problem $(1,4)$ as well as the related problems corresponding to the prescribed constant wall temperature and prescribed variable heat flux boundary conditions (2) and (3) share the very same mathematical core which is the conditional initial value problem $(12,13)$. The latter problem can easily be solved by standard numerical methods and its solution yields the characteristic curve $S=S(\lambda)$ plotted in Fig. 1.

(2) Every velocity solution of one of the three problems specified by some point $(\lambda, S)$ of the characteristic curve $S=S(\lambda)$, at the same time is a solution of the other two boundary value problems so that in the characteristic plane $(\lambda, S)$ the three problems become identical. As a direct consequence, the stability properties of the dual solutions found by Merkin (1985) in the prescribed constant wall temperature problem $(1,2)$ are valid in all three cases. Namely, the upper branch solutions are always linearly stable and the lower branch ones are unstable.

(3) Specific differences among the three boundary value problems occur only on the second level of consideration, i.e., when their common characteristic curve $S=S(\lambda)$ is mapped on the parameter plane $\left(\gamma, \varepsilon_{3}\right)$ in case of the convective problem $(1,4)$, and on the linear domains of the mixed convection parameters $\varepsilon_{1}$ and $\varepsilon_{2}$ in case of the other two problems. 
(4) In case of the prescribed wall temperature and of the prescribed heat flux problem, to every point of the characteristic curve $S=S(\lambda)$ there corresponds a unique value of the respective mixed convection parameter, so that the associated flow states $(\lambda, S)$ are nondegenerate with respect to $\varepsilon_{1}$ and $\varepsilon_{2}$. In the convective problem $(1,4)$, however, every point $(\lambda, S) \neq(1,0)$ of the characteristic curve is associated with a continuous infinity of values of the governing parameters $\left(\gamma, \varepsilon_{3}\right)$ satisfying the equation $S=-\gamma\left(1+\varepsilon_{3}-\lambda\right)$ so that every flow state $(\lambda, S) \neq(1,0)$ is infinitely degenerate with respect to $\left(\gamma, \varepsilon_{3}\right)$. Accordingly, to every point $(\lambda, S) \neq(1,0)$ of the characteristic curve $S=S(\lambda)$ there corresponds a whole curve $\varepsilon_{3}=\varepsilon_{3}(\gamma)$ described by Eq. (17) which is the trajectory of the flow state $(\lambda, S)$ in the parameter plane $\left(\gamma, \varepsilon_{3}\right)$ (Fig. 5).

(5) The Blasius bound $\left(f^{\prime}(0), f^{\prime \prime}(0)\right)=(0,0.4696)$ and the Steinheuer $\left(f^{\prime}(0), f^{\prime \prime}(0)\right)=$ $(-0.3541,0.2195)$ of the characteristic curve $S=S(\lambda)$ represent absolute upper and lower bounds of the solution domain with respect to the wall shear stress $f^{\prime \prime}(0)$ and the flow velocity at the wall $f^{\prime}(0)$, respectively. These points of the characteristic curve migrate in the parameter plane $\left(\varepsilon_{3}, \gamma\right)$ along the trajectories $\varepsilon_{3}=\varepsilon_{3}(\gamma)$ given by Eqs. (20) and (21) so that magnitudes $f_{\max }^{\prime \prime}(0)=0.4696$ and $f_{\min }^{\prime}(0)=-0.3541$ remain unchanged (Fig. 7a, b).

(6) The lower bound $\varepsilon_{3 \text {, crit }}(\gamma)$ of the solution domain with respect $\varepsilon_{3}$ corresponds to the value of the Biot number $\gamma$ for which the straight line $S=-\gamma\left(1+\varepsilon_{3}-\lambda\right)$ associated with the convective boundary condition (4) becomes tangent to the characteristic curve $S=S(\lambda)$. As $\gamma$ decreases from $+\infty$ to $0, \varepsilon_{3}$, crit $(\gamma)$ moves from the Steinheuer bound $\varepsilon_{3, \text { crit }}(\infty)=-1.3541$ toward $-\infty$ (Table 1; Fig. 6).

(7) To every point $(\lambda, S) \neq(1,0)$ of the characteristic curve $S=S(\lambda)$ there corresponds in the parameter plane $\left(\varepsilon_{3}, \gamma\right)$ a continuous infinity of distinguishable temperature solutions given by Eq. (22). Thus for the temperature solutions the infinite degeneracy of the flow solution $(\lambda, S)$ becomes lifted (Fig. 8). The wall temperature and its gradient can be calculated explicitly with aid of the simple relationships (23) (see also Fig. 9).

(8) Therefore, we may conclude that once the characteristic curve $S=S(\lambda)$ of the conditional initial value problem $(12,13)$ plotted in Fig. 1 is known, the three differential boundary value problems $(1,2),(1,3)$, and $(1,4)$ reduce to simple geometrical mapping problems of the characteristic curve $S=S(\lambda)$ on the respective parameter domains (Figs. 2, 3, and 4). In case of the convective boundary condition, the reduction consist of a mapping of the curve $S=S(\lambda)$ on the parameter plane $\left(\varepsilon_{3}, \gamma\right)$ by means of the transformation $S=-\gamma\left(1+\varepsilon_{3}-\lambda\right)$.

\section{References}

Aziz, A.: A similarity solution for laminar thermal boundary layer over a flat plate with a convective surface boundary condition. Commun. Nonlinear Sci. Numer. Simul. 14, 1064-1068 (2009)

Cortell, Bataller R.: Radiation effects for the Blasius and Sakiadis flows with convective surface boundary condition. Appl. Math. Comput. 206, 832-840 (2008)

Ishak, A.: Similarity solutions for flow and heat transfer over a permeable surface with convective boundary condition. Appl. Math. Comput. 217, 837-842 (2010)

Klemp, J.B., Acrivos, A.A.: A method for integrating the boundary-layer equations through a region of reverse flow. J. Fluid Mech. 53, 177-199 (1972)

Klemp, J.B., Acrivos, A.A.: A moving-wall boundary layer with reverse flow. J. Fluid Mech. 76, 363-381 (1976)

Lok, Y.Y., Merkin, J.H., Pop, I.: Mixed convection boundary-layer flow over a vertical surface embedded in a porous material subject to a convective boundary condition. Transp. Porous Media. doi:10.1007/ s11242-013-0153. Online First, 26 March 2013 
Magyari, E.: Comment on "A similarity solution for laminar thermal boundary layer over a flat plate with a convective surface boundary condition" by A. Aziz, Commun. Nonlinear Sci. Numer. Simul. 2009; 14:1064-1068. Commun. Nonlinear Sci. Numer. Simul. 16, 599-601 (2011)

Makinde, O.D., Aziz, A.: MHD mixed convection from a vertical plate embedded in a porous medium with a convective boundary condition. Int. J. Therm. Sci. 49, 1813-1820 (2010)

Makinde, O.D., Olanrewaju, P.O.: Buoyancy effects on thermal boundary layer over vertical plate with a convective surface boundary condition. J. Fluids Eng. 132, 044502-1-4 (2010)

Merkin, J.H.: Mixed convection boundary layer flow on a vertical surface in a saturated porous medium. J. Eng. Math. 14, 301-313 (1980)

Merkin, J.H.: On dual solutions occurring in mixed convection in a porous medium. J. Eng. Math. 20, 171-179 (1985)

Merkin, J.H., Pop, I.: The forced convection flow of a uniform streamover a flat surface with a convective surface boundary condition. Commun. Nonlinear Sci. Numer. Simul. 16, 3602-3609 (2011)

Steinheuer, J.: Die Lösung der Blasiusschen Grenzschichtdifferentialgleichung, Abhandlg. Braunschw. Wiss. Ges. 20, 96-125 (1968)

Uddin, J., Khan, W.A., Ismail, A.I.: Free convection boundary layer flow from a heated upward facing flat plate embedded in a porous medium filled by a nanofluid with convective boundary condition. Transp. Porous Media 92, 867-881 (2012) 